\title{
Catalytic Asymmetric Cyanosilylation of Ketones by a Chiral Amino Acid Salt
}

\author{
Xiaohua Liu, Bo Qin, Xin Zhou, Bin He, and Xiaoming Feng* \\ Key Laboratory of Green Chemistry \& Technology (Sichuan University), Ministry of Education, College \\ of Chemistry, Sichuan University, Chengdu 610064, P. R. China
}

\section{Supplementary Materials}

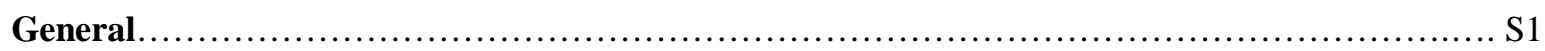

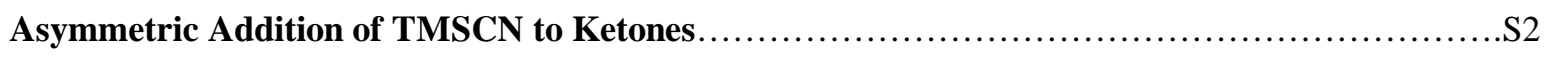

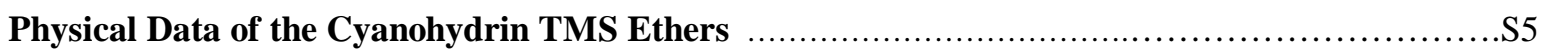

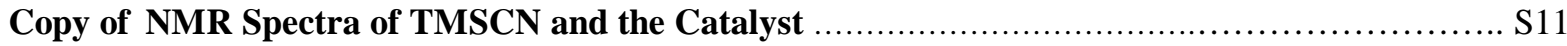

General. ${ }^{1}$ H NMR spectra were recorded on a Varian Unity INOVA 400 (400 MHz) or on Bruker instruments (600, $300 \mathrm{MHz}$ ). Chemical shifts are reported in ppm from tetramethylsilane with the solvent resonance as the internal standard $\left(\mathrm{CDCl}_{3}, \delta=7.26\right)$. Data are reported as follows: chemical shift, multiplicity $(\mathrm{s}=$ singlet, $\mathrm{d}=$ doublet, $\mathrm{t}=$ triplet, $\mathrm{m}=$ multiplet), coupling constants $(\mathrm{Hz})$, integration, and assignment. ${ }^{13} \mathrm{C}$ NMR data were collected on a Varian Unity INOVA 400 (100 MHz) or on Bruker instruments (150, $75 \mathrm{MHz})$ with complete proton decoupling. Chemical shifts are reported in ppm from the tetramethylsilane with the solvent resonance as internal standard $\left(\mathrm{CDCl}_{3}, \delta=77.0\right) .{ }^{29} \mathrm{Si} \mathrm{NMR}$ spectra were recorded on Bruker instruments $(300 \mathrm{MHz})$. Elemental analyses were performed on a Carlo-1160 apparatus. Enantiomer ratios were determined by chiral GC analysis on Varian Chirasil DEX CB or by chiral HPLC analysis on Daicel Chiralcel OD/OJ in comparison with the authentic racemates. Optical rotation data were recorded on Perkin-Elmer Polarimeter-341. All ketones, TMSCN and amino acids were purchased from Acros, Aldrich and Fluka. Solvents were purified by usual methods. 


\section{Catalyst Preparation.}

Organic Salts Preparation: Organic salts were prepared by adding 1 equiv of alkali metal hydroxide to the organic acid in methanol at $0{ }^{\circ} \mathrm{C}$ and stirring at $25{ }^{\circ} \mathrm{C}$ for $1-3 \mathrm{~h}$. After the solvent was evaporated, the salts were dried at $25{ }^{\circ} \mathrm{C}$ in vacuo. The salts thus obtained were used without futher purification.

$\boldsymbol{L}$-phenylglycine sodium salt. Anal Calcd for $\mathrm{C}_{8} \mathrm{H}_{8} \mathrm{NNaO}_{2} .{ }^{1}{ }_{2} \mathrm{H}_{2} \mathrm{O}: \mathrm{C}, 52.26 ; \mathrm{N}, 7.61 ; \mathrm{H}, 4.38$. Found: C, 52.05; N, 7.58; H, 4.40 .

\section{Preparation of optically active cyanohydrin trimethylsilyl ethers 3}

Method A: (footnote a in Talbe 1) To a mixture of ketone $(0.5 \mathrm{mmol})$ and organic salt $2(0.15 \mathrm{mmol})$ in $0.5 \mathrm{~mL}$ of solvent was added trimethylsiyl cyanide (1.2 equiv) and $0.5 \mathrm{~mL}$ solvent at $-20{ }^{\circ} \mathrm{C}$. The reaction was monitored by TLC. After the reaction, organic salts precipitated and was filtrated from the reaction mixture and the residues were purified by flash chromatography to give the pure product with ethyl ether/petroleum ether $(1 / 100, \mathrm{~V} / \mathrm{V})$ as the eluent.

Method B: (footnote $b$ in Table 1) To a solution of $L$-phenylglycine sodium salt in $0.5 \mathrm{~mL}$ of solvent was added trimethylsiyl cyanide $\left(1.2\right.$ equiv) at $-20{ }^{\circ} \mathrm{C}$, and the mixture was stirred at $30{ }^{\circ} \mathrm{C}$ for $1.0 \mathrm{~h}$. And then ketone $(0.5 \mathrm{mmol})$ in $0.5 \mathrm{~mL}$ THF was dropwise added to the solution under the reaction condition indicated in Table 2 and monitored by TLC. After warming to room temperature, $L$-phenylglycine sodium salt was filtrated form the reaction mixture and the residues concentrated and put on a silica gel column to give the pure product with ethyl ether/petroleum ether (1/100, $\mathrm{V} / \mathrm{V})$ as the eluent.

Method C: (footnote $c$ in Table 1) $L$-Phenylglycine $(22.7 \mathrm{mg}, 0.15 \mathrm{mmol})$ was stirred with $\mathrm{NaOH}(6.0 \mathrm{mg}, 0.15 \mathrm{mmol})$ in dry THF $(0.3 \mathrm{~mL})$ at $25{ }^{\circ} \mathrm{C}$ for $0.5 \mathrm{~h}$ under $\mathrm{N}_{2}$ atmosphere. To the resulted catalyst, TMSCN and THF $(0.2 \mathrm{~mL})$ was added at $-20{ }^{\circ} \mathrm{C}$. After stirring for $1.0 \mathrm{~h}$ at $30{ }^{\circ} \mathrm{C}$, ketone in THF was dropwise added. Then the reaction mixture was stirred under the reaction condition indicated in Table 2 of the communication and monitored by TLC. After warming to room temperature, $L$-phenylglycine sodium salt was filtrated form the reaction mixture and the residues concentrated and put on a silica gel column to give the pure product with ethyl ether/petroleum ether $(1 / 100, \mathrm{~V} / \mathrm{V})$ as the eluent.

Method D: (footnote $d$ in Table 1) To a solution of $L$-phenylglycine sodium salt $2 \mathbf{f}^{1} /{ }_{2} \mathbf{H}_{2} \mathbf{O}$ in $0.5 \mathrm{~mL}$ of THF was added trimethylsiyl cyanide $\left(1.5\right.$ equiv) at $-20{ }^{\circ} \mathrm{C}$, and the mixture was stirred at $30{ }^{\circ} \mathrm{C}$ for $1.0 \mathrm{~h}$. And then ketone $(0.5 \mathrm{mmol})$ was charged sequentially at $-45{ }^{\circ} \mathrm{C}$ and stirred for $15 \mathrm{~min} .{ }^{i} \mathrm{PrOH}(0.25 \mathrm{mmol})$ and $0.5 \mathrm{~mL}$ THF were then added via syringe. The reaction stirred at $-45{ }^{\circ} \mathrm{C}$ for certain time and monitored by TLC. After warming to room temperature, $L-$ phenylglycine sodium salt was filtrated form the reaction mixture and the residues concentrated and put on a silica gel column to give the pure product with ethyl ether/petroleum ether $(1 / 100, \mathrm{~V} / \mathrm{V})$ as the eluent. 
Note: 0.3 equiv methanol could also be added at the initial stage before TMSCN was syringed in Method D and the same results could be obtained. The tolerance of the catalyst enhanced greatly by this method. It may due to the optimal capacity of protonic impurity in the catalyst is within the range of 0.5-1.0 equiv of the catalyst $\mathbf{2 f}$, and the methanol could play the same role as water at the first stage of the reaction.

\section{Optimization Studies.}

Solvent effects were studied with the results summarized in Table 1. THF was the most favorable solvent for enantioselectivity.

Table I Effects of solvent on the asymmetric addition of TMSCN to acetophenone. ${ }^{\mathrm{a}}$

\begin{tabular}{cccc}
\hline solvent & $\begin{array}{c}\text { Loading } \\
(\mathrm{mol} \%)\end{array}$ & ${\text { Yield }(\%)^{b}}^{b}$ & $\mathrm{Ee}(\%)^{c}$ \\
\hline $\mathrm{Et}_{2} \mathrm{O}$ & 30 & 45 & 44 \\
$\mathrm{CH}_{2} \mathrm{Cl}_{2}$ & 30 & 51 & 36 \\
$\mathrm{CHCl}_{3}$ & 30 & 84 & 29 \\
Toluene & 30 & 64 & 31 \\
Benzene & 30 & 70 & 26 \\
Acetonitrile & 30 & 52 & 20 \\
1,4 -Dioxane & 30 & 71 & 34 \\
$t$-BuOMe & 30 & 55 & 26 \\
THF & 30 & 50 & 54 \\
\hline
\end{tabular}

${ }^{a}$ The reactions were carried out on a $0.5 \mathrm{mmol}$ scale with $30 \mathrm{~mol} \%$ catalyst $2 \mathbf{f}$ and 1.2 eq TMSCN at-20 ${ }^{\circ} \mathrm{C}$ by Method A mentioned above. ${ }^{b}$ Isolated yield. ${ }^{c}$ Determined by chiral GC analysis on Chiasil DEX CB.

Table II. Effects of catalyst loading on the asymmetric addition of TMSCN to acetophenone. ${ }^{a}$

\begin{tabular}{cccc}
\hline $\begin{array}{c}\text { Catalyst loading } \\
(\text { mol \% })\end{array}$ & $\begin{array}{c}\text { concentration } \\
(\mathrm{M})\end{array}$ & ${\text { Yield }(\%)^{\mathrm{b}}}^{\mathrm{Ee}(\%)^{\mathrm{c}}}$ \\
\hline 5 & 0.5 & 31 & 0 \\
10 & 0.5 & 60 & 0 \\
10 & 1.5 & 65 & 11 \\
20 & 0.5 & 66 & 38 \\
20 & 1.0 & 68 & 56 \\
20 & 1.5 & 72 & 61 \\
20 & 2.0 & 72 & 61 \\
25 & 0.5 & 73 & 60 \\
30 & 0.2 & 24 & 11 \\
30 & 0.4 & 42 & 79 \\
30 & 0.5 & 60 & 81 \\
30 & 1.0 & 52 & 82 \\
\hline
\end{tabular}




\begin{tabular}{llll}
\hline 35 & 0.5 & 46 & 83 \\
40 & 0.5 & 21 & 83 \\
50 & 0.5 & 18 & 82 \\
\hline
\end{tabular}

${ }^{a}$ The reactions were carried out with catalyst $\mathbf{2 f}$ and 1.2 eq TMSCN in THF at- $20{ }^{\circ} \mathrm{C}$ by Method $\mathbf{C}$ mentioned above. ${ }^{b}$ Isolated yield. ${ }^{c}$ Determined by chiral GC analysis on Chiasil DEX CB.

The effects of catalyst loading were systematically studied. The results, described in Table 2 , indicated that the enantioselectivity and yield were influenced by the amount of the chiral amino acid salt. The best result was obtained when $30 \mathrm{~mol} \%$ catalyst loading was used. Lowering the catalyst loading to less then $10 \mathrm{~mol} \%$ led to dramatic drops in ee value with racemic products and the reaction solution appeared a semitransparent state nor a heterogeneous state. On increasing of the catalyst loading from 30 to $50 \mathrm{~mol} \%$, the yield was sharply decreased for the water contained in the catalyst reacted with TMSCN and relayed the addition of TMSCN to acetophenone and a small amount of undetermined by-product was formed under this reaction condition.

In order to survey the role of the amino group of catalyst $\mathbf{2 f}$, modified amino acid salts were used as catalysts for cyanosilylation of acetophenone. From Table 4, we can see that any modification of the primary amine lower the ee values of the products.

Table III. Structral effect of the catalysts on the asymmetric cyanosilylation of acetophenone ${ }^{\mathrm{a}}$

\begin{tabular}{lccc}
\hline Entry & Time(h) & yield (\%) & ee (\%) \\
\hline 1 & 68 & 40 & 2 \\
\hline
\end{tabular}

${ }^{a}$ The reactions were carried out on a $0.5 \mathrm{mmol}$ scale with $30 \mathrm{~mol} \%$ catalyst loading and 1.2 eq TMSCN at- $20{ }^{\circ} \mathrm{C}$ by Method A mentioned above. ${ }^{b}$ isolated yield. ${ }^{c}$ determined by chiral GC analysis on Chiasil DEX CB.

Table IV. Effects of additive and experiment procedure on the asymmetric addition of TMSCN to acetophenone ${ }^{\mathrm{a}}$

\begin{tabular}{cccccccc}
\hline Entry & catalyst & procedure & $\begin{array}{c}\text { TMSCN } \\
(\text { eq. })\end{array}$ & additive & $\begin{array}{c}\text { temp } \\
\left({ }^{\circ} \mathrm{C}\right)\end{array}$ & $\begin{array}{c}\text { yield } \\
(\%)\end{array}$ & $\begin{array}{c}\text { ee } \\
(\%)\end{array}$ \\
\hline 1 & $\mathbf{2 f}$ & $\mathrm{a}$ & 1.2 & -- & -20 & 58 & 5.2 \\
\hline
\end{tabular}




\begin{tabular}{cccccccc}
\hline 2 & $\mathbf{2 f} \cdot 1 / 2 \mathbf{H}_{2} \mathbf{O}$ & $\mathrm{a}$ & 1.2 & -- & -20 & 50 & 51 \\
3 & L-phenglycine $+\mathrm{NaOH}$ & $\mathrm{a}$ & 1.2 & -- & -20 & 70 & 54 \\
4 & L-phenglycine $+\mathrm{NaOH}$ & $\mathrm{c}$ & 1.2 & -- & -20 & 68 & 80 \\
5 & L-phenglycine $+\mathrm{NaOH}$ & $\mathrm{b}$ & 1.2 & -- & -45 & 55 & 53 \\
6 & L-phenglycine $+\mathrm{NaOH}$ & $\mathrm{c}$ & 1.2 & -- & -45 & 64 & 94 \\
7 & L-phenglycine $+\mathrm{NaOH}$ & $\mathrm{c}$ & 1.5 & -- & -45 & 84 & 92 \\
8 & $\mathbf{2 f} \cdot 1 / 2 \mathbf{H}_{\mathbf{2}} \mathbf{O}$ & $\mathrm{d}$ & 2.0 & $\mathrm{H}_{2} \mathrm{O}(0.5$ eq. $)$ & -20 & 97 & 64 \\
9 & $\mathbf{2 f} \cdot 1 / 2 \mathbf{H}_{2} \mathbf{O}$ & $\mathrm{d}$ & 1.5 & $\mathrm{CH}_{3} \mathrm{OH}(0.5$ eq. $)$ & -45 & 85 & 93 \\
10 & $\mathbf{2 f} \cdot 1 / 2 \mathbf{H}_{\mathbf{2}} \mathbf{O}$ & $\mathrm{d}$ & 1.5 & ${ }^{i} \mathrm{PrOH}(0.5$ eq. $)$ & -45 & 96 & 94 \\
11 & $\mathbf{2 f} \cdot 1 / 2 \mathbf{H}_{\mathbf{2}} \mathbf{O}$ & $\mathrm{e}$ & 1.5 & ${ }^{i} \mathrm{PrOH}(0.5$ eq. $)$ & -45 & 95 & 94 \\
\hline
\end{tabular}

${ }^{a}$ Procedure a: the reactions were carried out by Method A mentioned above ; Procedure b: $L$-Phenylglycine $(22.7 \mathrm{mg}, 0.15 \mathrm{mmol}) \mathrm{was}$ stirred with $\mathrm{NaOH}(6.0 \mathrm{mg}, 0.15 \mathrm{mmol})$ in dry THF $(0.3 \mathrm{~mL})$ at $25{ }^{\circ} \mathrm{C}$ for $0.5 \mathrm{~h}$ under $\mathrm{N}_{2}$ atmosphere. To the resulted catalyst, acetophenone $(0.5 \mathrm{mmol})$ and THF $(0.2 \mathrm{~mL})$ was added at $-20{ }^{\circ} \mathrm{C}$ and stirred at $30^{\circ} \mathrm{C}$ for an hour, and then added TMSCN (1.2 eq) and 0.5 $\mathrm{mL}$ THF at $-45^{\circ} \mathrm{C}$; Procedure c: the reactions were carried out by Method $\mathbf{C}$ mentioned above; Procedure d: the reaction was carried out by Method $\mathbf{D}$ and ${ }^{i} \mathrm{PrOH}$ was replace by the corresponding additives; Procedure e: To a solution of L-phenylglycine sodium salt and 0.3 equiv $\mathrm{CH}_{3} \mathrm{OH}$ in $0.5 \mathrm{~mL}$ of THF was added trimethylsiyl cyanide $\left(1.5\right.$ equiv) at $-20{ }^{\circ} \mathrm{C}$, and the mixture was stirred at $30{ }^{\circ} \mathrm{C}$ for $1.0 \mathrm{~h}$. And then ketone $(0.5 \mathrm{mmol})$ was charged sequentially at $-45^{\circ} \mathrm{C}$ and stirred for $15 \mathrm{~min}$. $i \mathrm{PrOH}(0.25 \mathrm{mmol})$ and $0.5 \mathrm{~mL}$ THF were then added via syringe. The reaction stirred at $-45^{\circ} \mathrm{C}$ for certain time and monitored by TLC. ${ }^{b}$ isolated yield; ${ }^{c}$ determined by chiral GC analysis on Chiasil DEX CB

One key feature associated with the use of $L$-phenylglycine sodium salt was the experimental procedure (Table 5). When the catalyst was stirred with TMSCN in THF for $1 \mathrm{~h}$ at $30{ }^{\circ} \mathrm{C}$ before acetophenone was added, the enantioselectivity was greatly improved from $54 \%$ to $80 \%$ ee at $-20{ }^{\circ} \mathrm{C}$ and up to $94 \%$ ee at a lower reaction temperature $\left(-45^{\circ} \mathrm{C}\right)$. A little moisture was crucial to retain enantioselectivity, and any attempt to remove water contained in the catalyst gave bad result (Table IV, entries 1-3). Howerver, introduction of alcohol increased catalytic activity and led to product formation in $96 \%$ yield and $94 \%$ ee (entries $7,9-10$ ) .

\section{Preparation of racemic samples for GC and HPLC analysis.}

To $30 \mathrm{~mol} \% \mathrm{~K}_{2} \mathrm{CO}_{3}$, ketone and $1.2 \mathrm{TMSCN}$ were dropwise added and stirred at room temperature for $24 \mathrm{~h}$. Then $\mathrm{K}_{2} \mathrm{CO}_{3}$ was filtrated form the reaction mixture and the residues was put on a silica gel column to give the pure product with ethyl ether/petroleum ether $(1 / 100, \mathrm{~V} / \mathrm{V})$ as the eluent.

\section{Physical Data of the Cyanohydrin TMS Ethers Reported in the communication.}

\section{2-trimethylsilyloxy-2-phenylpropanenitrile (3a)}

96\% yield, 94\% ee. ${ }^{1} \mathrm{H}$ NMR (300 MHz, $\left.\mathrm{CDCl}_{3}\right): \delta=0.19(\mathrm{~s}, 9 \mathrm{H}), 1.87(\mathrm{~s}, 3 \mathrm{H}), 7.38-7.58(\mathrm{~m}, 5 \mathrm{H}) \mathrm{ppm}$. GC analysis (Varian, CP-Chirasil DEX CB, $\left.0.25 \mathrm{~mm} \times 25 \mathrm{~m}, 105{ }^{\circ} \mathrm{C}, 8 \mathrm{psi}\right): t_{\mathrm{r}}($ major $)=22.0 \mathrm{~min}, t_{\mathrm{r}}(\operatorname{minor})=21.3 \mathrm{~min} .[\alpha]_{\mathrm{D}}^{25}+22.0$ 
( $c=0.204$ in $\mathrm{CH}_{2} \mathrm{Cl}_{2}, 94 \%$ ee). [lit. [ $\left.\alpha\right]_{\mathrm{D}}{ }^{24}-21.9\left(c=1.18\right.$ in $\mathrm{CH}_{2} \mathrm{Cl}_{2}, 93 \%$ ee)]; Fuerst, D. E.; Jacobsen, E. N. J. Am.

Chem. Soc. 2005, 127, 8964-8965.
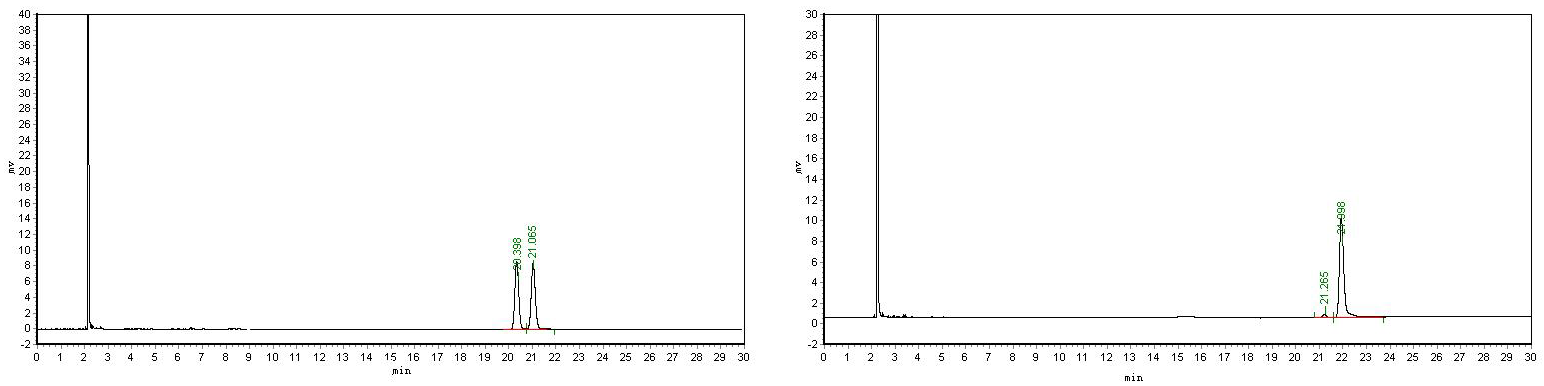

\section{2-trimethylsilyloxy-2-(4'-methoxylphenyl)propanenitrile ( 3b)}

$81 \%$ yield, $92 \%$ ee. ${ }^{1} \mathrm{H}$ NMR $\left(400 \mathrm{MHz}, \mathrm{CDCl}_{3}\right): \delta=0.19(\mathrm{~s}, 9 \mathrm{H}), 1.87(\mathrm{~s}, 3 \mathrm{H}), 3.85(\mathrm{~s}, 3 \mathrm{H}), 6.93(\mathrm{~m}, 2 \mathrm{H}), 7.49(\mathrm{~m}$, 2H) ppm. ${ }^{13} \mathrm{C}$ NMR $\left(100 \mathrm{MHz}, \mathrm{CDCl}_{3}\right): \delta=33.41,55.33,71.28,113.89,121.81,126.06,134.04,159.80 \mathrm{ppm} . \mathrm{GC}$ analysis (Varian, CP-Chirasil DEX CB, 0.25mm x $\left.25 \mathrm{~m}, 105{ }^{\circ} \mathrm{C}, 8 \mathrm{psi}\right): t_{\mathrm{r}}($ major$)=86.4 \mathrm{~min}, t_{\mathrm{r}}(\operatorname{minor})=85.2 \mathrm{~min}$. $[\alpha]_{\mathrm{D}}^{25}+22.9\left(c=0.188\right.$ in $\mathrm{CH}_{2} \mathrm{Cl}_{2}, 92 \%$ ee $)$. [lit. $[\alpha]_{\mathrm{D}}^{20}+22.6\left(c=1.09\right.$ in $\mathrm{CHCl}_{3}, 91 \%$ ee $\left.)\right]$; Deng, H.; Isler, M. P.; Snapper, M. L.; Hoveyda, A. H. Angew. Chem. Int. Ed. 2002, 41, 1009-1021.
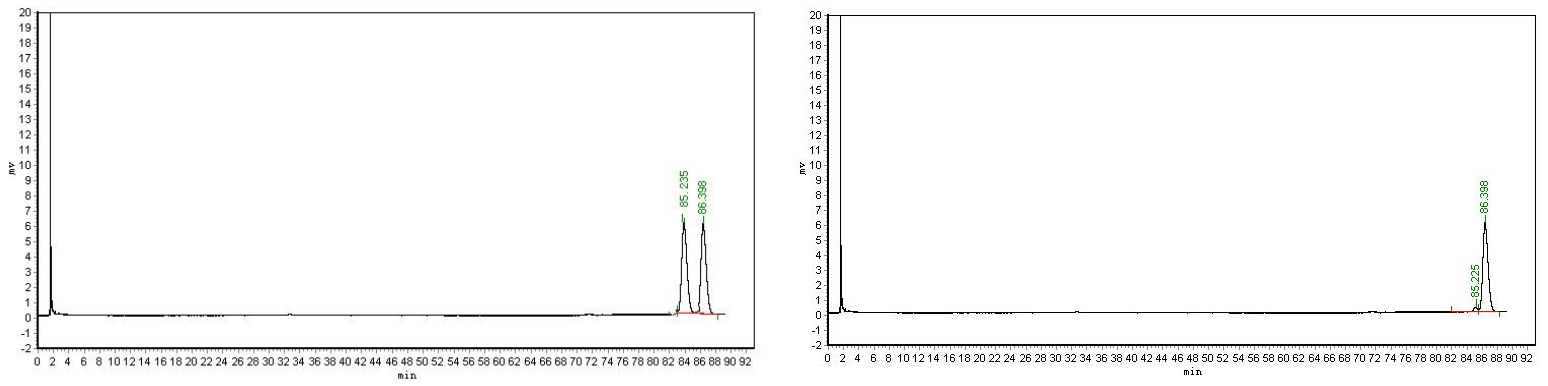

\section{2-trimethylsilyloxy-2(4'-methylphenyl)propanenitrile (3c)}

75\% yield, $97 \%$ ee. ${ }^{1} \mathrm{H}$ NMR $\left(400 \mathrm{MHz}, \mathrm{CDCl}_{3}\right): \delta=0.16(\mathrm{~s}, 9 \mathrm{H}), 1.84(\mathrm{~s}, 3 \mathrm{H}), 2.36(\mathrm{~s}, 3 \mathrm{H}), 7.21(\mathrm{~m}, 2 \mathrm{H}), 7.43(\mathrm{~m}, 2 \mathrm{H})$ ppm. $\mathrm{C}_{13} \mathrm{H}_{19}$ NOSi: C 66.90, H 8.21, N 6.00; found C 66.78, H 8.03, N 6.39. GC analysis (Varian, CP-Chirasil DEX CB, $\left.0.25 \mathrm{~mm} \times 25 \mathrm{~m}, 105^{\circ} \mathrm{C}, 8 \mathrm{psi}\right): t_{\mathrm{r}}$ (major) $=38.4 \mathrm{~min}, t_{\mathrm{r}}($ minor $)=37.8 \mathrm{~min} .[\alpha]_{\mathrm{D}}^{25}+24.3\left(c=0.192\right.$ in $\mathrm{CH}_{2} \mathrm{Cl}_{2}, 97 \%$ ee $)$. [lit. $[\alpha]_{\mathrm{D}}^{25}+22.1\left(c=2.44\right.$ in $\mathrm{CH}_{2} \mathrm{Cl}_{2}, 92 \%$ ee)]; Chen, F. X.; Zhou, H.; Liu, X. H.; Qin, B.; Feng, X. M.; Zhang, G. L.; Jiang, Y. Z. Chem. Eur. J. 2004, 10, 4790-4797.
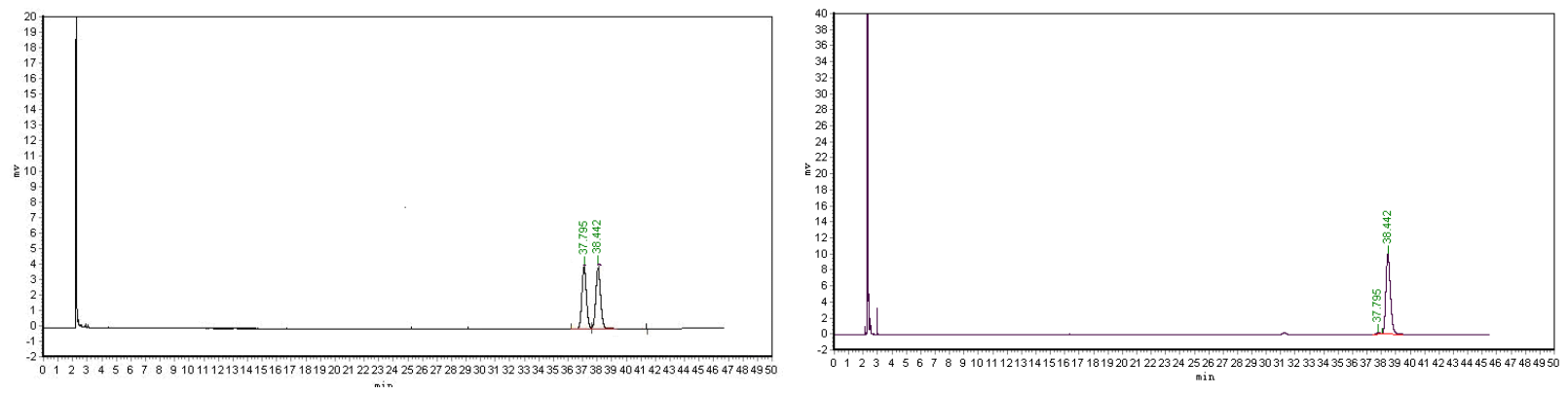


\section{2-trimethylsilyloxy-2(4'-fluorophenyl)propanenitrile (3d)}

90\% yield, $92 \%$ ee. ${ }^{1} \mathrm{H}$ NMR $\left(400 \mathrm{MHz}, \mathrm{CDCl}_{3}\right): \delta=0.18(\mathrm{~s}, 9 \mathrm{H}), 1.84(\mathrm{~s}, 3 \mathrm{H}), 7.08(\mathrm{~m}, 2 \mathrm{H}), 7.52(\mathrm{~m}, 2 \mathrm{H}) \mathrm{ppm} .{ }^{13} \mathrm{C}$ $\operatorname{NMR}\left(100 \mathrm{MHz}, \mathrm{CDCl}_{3}\right): \delta=1.0,33.5,71.0,115.6\left(\mathrm{~d},{ }^{2} J_{\mathrm{CCF}}=21.9 \mathrm{~Hz}\right), 121.4,126.5\left(\mathrm{~d},{ }^{3} J_{\mathrm{CCCF}}=8.5 \mathrm{~Hz}\right), 138.0$, 162.2 $\left(\mathrm{d},{ }^{1} J_{\mathrm{CF}}=246.4 \mathrm{~Hz}\right) \mathrm{ppm}$. GC analysis (Varian, CP-Chirasil DEX CB, 0.25mm x 25m ,115 $\left.{ }^{\circ} \mathrm{C}, 8 \mathrm{psi}\right): t_{\mathrm{r}}($ major $)=$ 19.1 min, $t_{\mathrm{r}}($ minor $)=18.3$ min. $[\alpha]_{\mathrm{D}}^{25}+17.8\left(c=0.212\right.$ in $\mathrm{CH}_{2} \mathrm{Cl}_{2}, 92 \%$ ee $)$. [lit. $[\alpha]_{\mathrm{D}}^{22}+17.6\left(c=2.7\right.$ in $\mathrm{CH}_{2} \mathrm{Cl}_{2}, 92 \%$ ee)]; Chen, F. X.; Zhou, H.; Liu, X. H.; Qin, B.; Feng, X. M.; Zhang, G. L.; Jiang, Y. Z. Chem. Eur. J. 2004, 10, 47904797.
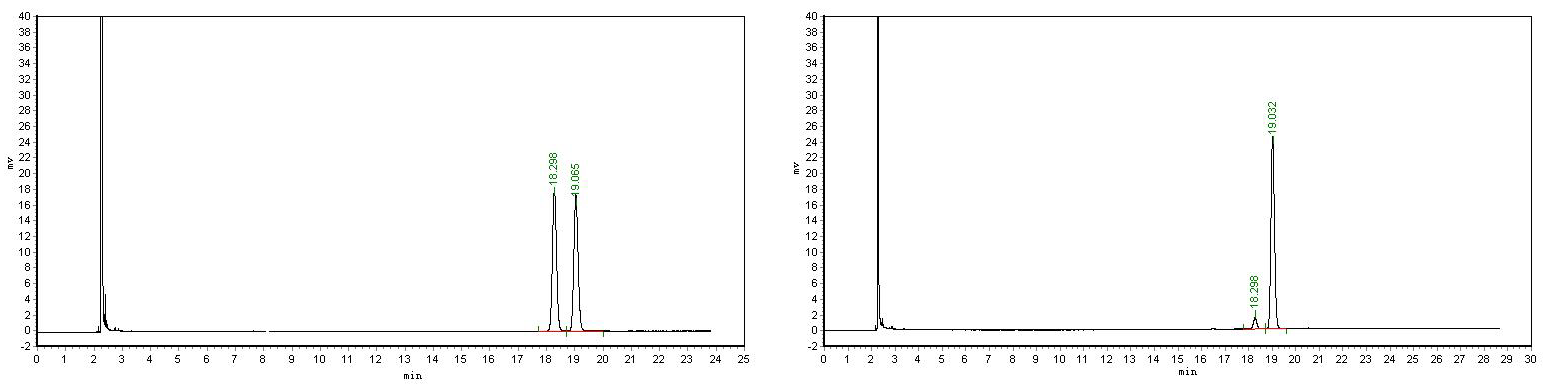

\section{2-trimethylsilyloxy-2(4'-chlorophenyl)propanenitrile (3e)}

$83 \%$ yield, $90 \%$ ee. ${ }^{1} \mathrm{H}$ NMR $\left(400 \mathrm{MHz}, \mathrm{CDCl}_{3}\right): \delta=0.19(\mathrm{~s}, 9 \mathrm{H}), 1.83(\mathrm{~s}, 3 \mathrm{H}), 7.38(\mathrm{~m}, 2 \mathrm{H}), 7.48(\mathrm{~m}, 2 \mathrm{H}) \mathrm{ppm}$. $\mathrm{C}_{12} \mathrm{ClH}_{16} \mathrm{NOSi}$ C 56.79, H 6.35, N 5.52; found C 56.82, H 6.41, N 5.93. GC analysis (Varian, CP-Chirasil DEX CB, $\left.0.25 \mathrm{~mm} \times 25 \mathrm{~m}, 110^{\circ} \mathrm{C}, 8 \mathrm{psi}\right): t_{\mathrm{r}}$ (major) $=53.6 \mathrm{~min}, t_{\mathrm{r}}($ minor $)=51.5 \mathrm{~min} .[\alpha]_{\mathrm{D}}^{25}+18.1\left(c=0.240\right.$ in $\mathrm{CH}_{2} \mathrm{Cl}_{2}, 90 \%$ ee $)$. [lit. $[\alpha]_{\mathrm{D}}^{25}+18.2\left(c=2.06\right.$ in $\mathrm{CH}_{2} \mathrm{Cl}_{2}, 90 \%$ ee)]; Chen, F. X.; Zhou, H.; Liu, X. H.; Qin, B.; Feng, X. M.; Zhang, G. L.; Jiang, Y. Z. Chem. Eur. J. 2004, 10, 4790-4797.
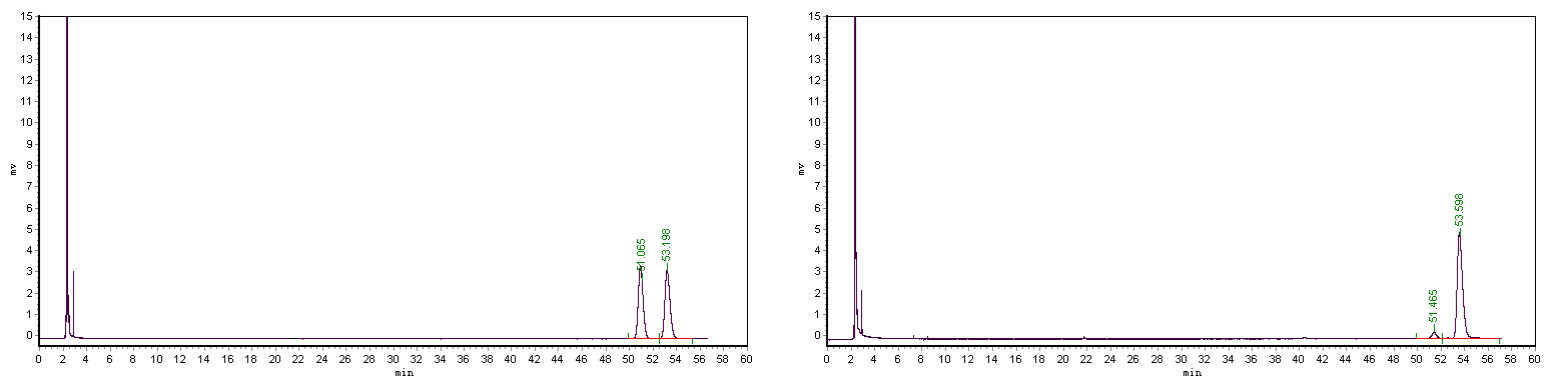

\section{2-trimethylsilyloxy-2(3'-chlorophenyl)propanenitrile (3f)}

$80 \%$ yield, $96 \%$ ee. ${ }^{1} \mathrm{H}$ NMR (300 MHz, $\left.\mathrm{CDCl}_{3}\right): \delta=0.22(\mathrm{~s}, 9 \mathrm{H}), 1.86(\mathrm{~s}, 3 \mathrm{H}), 7.34-7.55(\mathrm{~m}, 4 \mathrm{H}) \mathrm{ppm} .{ }^{13} \mathrm{C}$ NMR $(75$ $\left.\mathrm{MHz}, \mathrm{CDCl}_{3}\right): \delta=1.0,33.4,70.9,121.0,122.7,124.8,128.8,129.9,134.6,144.0$ ppm. $\mathrm{C}_{12} \mathrm{ClH}_{16} \mathrm{NOSi}_{\mathrm{C}} \mathrm{C} 56.79, \mathrm{H}$ 6.35, N 5.52; found C 56.61, H 6.39, N 5.90. GC analysis (Varian, CP-Chirasil DEX CB, $0.25 \mathrm{~mm}$ x 25m , $105{ }^{\circ} \mathrm{C}, 8$ psi): $t_{\mathrm{r}}($ major $)=49.9 \min , t_{\mathrm{r}}($ minor $)=49.0 \mathrm{~min} .[\alpha]_{\mathrm{D}}{ }^{25}+25.0\left(c=0.244\right.$ in $\mathrm{CH}_{2} \mathrm{Cl}_{2}, 96 \%$ ee $)$. [lit. $[\alpha]_{\mathrm{D}}{ }^{26}+19.6(c=2.88$ in $\mathrm{CH}_{2} \mathrm{Cl}_{2}$, 90\% ee)]; Chen, F. X.; Zhou, H.; Liu, X. H.; Qin, B.; Feng, X. M.; Zhang, G. L.; Jiang, Y. Z. Chem. Eur. J. 

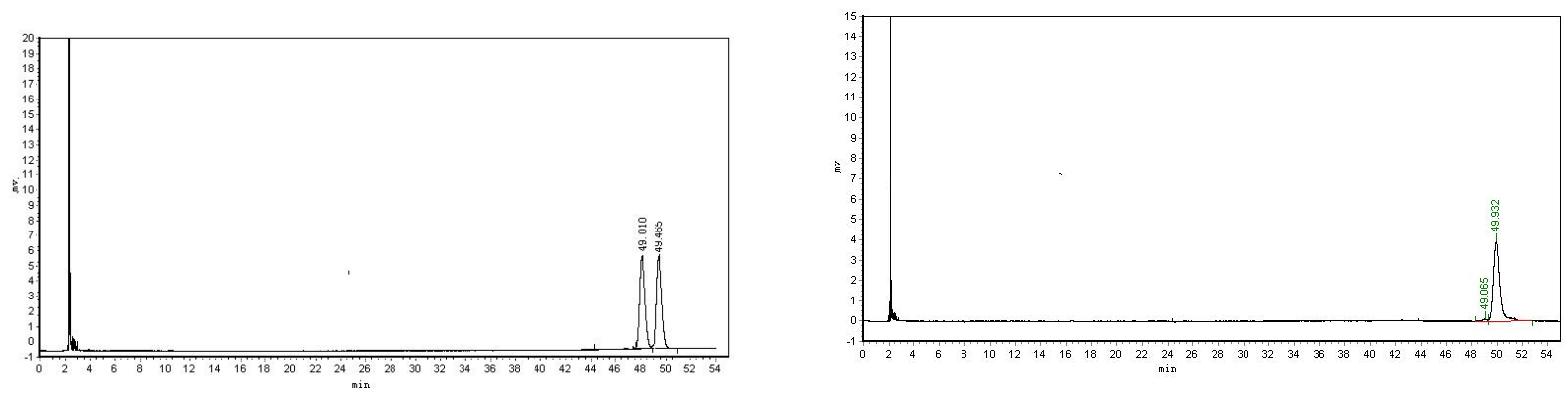

\section{2-trimethylsilyloxy-2(2'-fluorophenyl)propanenitrile (3g)}

77\% yield, $90 \%$ ee. ${ }^{1} \mathrm{H}$ NMR $\left(400 \mathrm{MHz}, \mathrm{CDCl}_{3}\right): \delta=0.26(\mathrm{~s}, 9 \mathrm{H}), 1.94(\mathrm{~s}, 3 \mathrm{H}), 7.07-7.12(\mathrm{~m}, 1 \mathrm{H}), 7.16-7.20(\mathrm{~m}, 1 \mathrm{H})$, 7.33-7.38 (m, 1H), 7.56-7.60 (m, 1H) ppm. ${ }^{13} \mathrm{C}$ NMR (100 MHz, $\left.\mathrm{CDCl}_{3}\right): \delta=1.0,30.8,68.4,116.5,120.6,124.2$, 126.7, 130.6, $159.4 \mathrm{ppm}$. GC analysis (Varian, CP-Chirasil DEX CB, 0.25mm x 25m ,105 ${ }^{\circ} \mathrm{C}, 8$ psi): $t_{\mathrm{r}}$ (major) $=25.6$ $\min , t_{\mathrm{r}}($ minor $)=25.0$ min. $[\alpha]_{\mathrm{D}}{ }^{25}+14.8\left(c=0.196\right.$ in $\mathrm{CH}_{2} \mathrm{Cl}_{2}, 90 \%$ ee $)$. [lit. $[\alpha]_{\mathrm{D}}{ }^{26}-12.7\left(c=1.18\right.$ in $\mathrm{CHCl}_{3}, 76 \%$ ee $\left.)\right]$; Chen, F. X.; Qin, B.; Feng, X. M.; Zhang, G. L.; Jiang, Y. Z. Tetrahedron 2004, 60, 10449-10460.
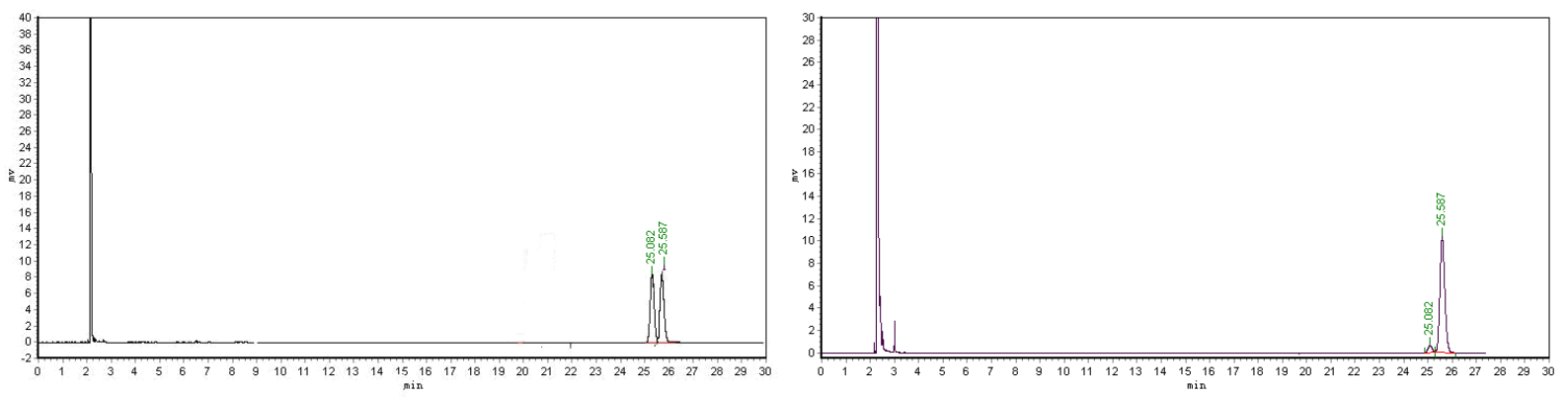

\section{2-trimethylsilyloxy- (1'-thiophene)-1-carbonitrile ( 3h)}

$58 \%$ yield, $92 \%$ ee $\left(84 \%\right.$ yield, $86 \%$ ee).${ }^{1} \mathrm{H}$ NMR $\left(300 \mathrm{MHz}, \mathrm{CDCl}_{3}\right): \delta=0.20(\mathrm{~s}, 9 \mathrm{H}), 2.00(\mathrm{~s}, 3 \mathrm{H}), 7.00(\mathrm{~m}, 1 \mathrm{H})$, 7.21-7.34 (m, 2H) ppm. ${ }^{13} \mathrm{C} \mathrm{NMR}\left(75 \mathrm{MHz}, \mathrm{CDCl}_{3}\right): \delta=0.78,33.40,68.24,120.82,124.70,125.97,126.61,146.27$ ppm. $\mathrm{C}_{10} \mathrm{H}_{15} \mathrm{NOSSi}$ : C 53.29, H 6.71, N 6.21; found C 53.48, H 6.94, N 6.43. GC analysis (Varian, CP-Chirasil DEX $\left.\mathrm{CB}, 0.25 \mathrm{~mm} \times 25 \mathrm{~m}, 110^{\circ} \mathrm{C}, 8 \mathrm{psi}\right): t_{\mathrm{r}}$ (major) $=22.7 \mathrm{~min}, t_{\mathrm{r}}($ minor $)=21.8 \mathrm{~min} . \quad[\alpha]_{\mathrm{D}}{ }^{25}+51.1\left(c=0.188\right.$ in $\mathrm{CH}_{2} \mathrm{Cl}_{2}, 92 \%$ ee), $[\alpha]_{\mathrm{D}}{ }^{25}+49.2\left(c=0.248\right.$ in $\mathrm{CH}_{2} \mathrm{Cl}_{2}, 86 \%$ ee $)$. [lit. $[\alpha]_{\mathrm{D}}^{22}-57.6\left(c=1.0\right.$ in $\mathrm{CHCl}_{3}, 97 \%$ ee $\left.)\right]$; Fuerst, D. E.; Jacobsen, E. N. J. Am. Chem. Soc. 2005, 127, 8964-8965.
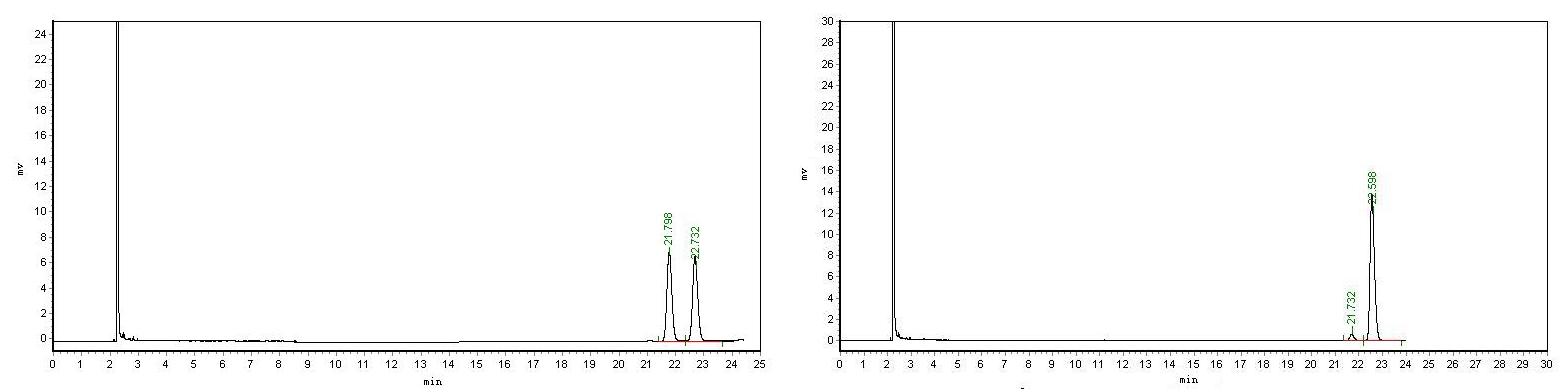


\section{2-trimethylsilyloxy-2-methyl-4-phenyl-3-butenenitrile (3i)}

96\% yield, $97 \%$ ee. ${ }^{1} \mathrm{H}$ NMR $\left(300 \mathrm{MHz}, \mathrm{CDCl}_{3}\right): \delta=0.27(\mathrm{~s}, 9 \mathrm{H}), 1.77(\mathrm{~s}, 3 \mathrm{H}), 6.15(\mathrm{~d}, J=15.9 \mathrm{~Hz}, 1 \mathrm{H}), 6.91(\mathrm{~d}, J$ $=15.9 \mathrm{~Hz}, 1 \mathrm{H}), 7.331-7.45(\mathrm{~m}, 5 \mathrm{H}) \mathrm{ppm} .{ }^{13} \mathrm{C} \mathrm{NMR}\left(75 \mathrm{MHz}, \mathrm{CDCl}_{3}\right): \delta=1.3,30.8,69.9,120.6,126.8,128.5,128.7$, 129.5, 130.9, $135.1 \mathrm{ppm}$. HPLC (Chiralcel OD, $i \mathrm{PrOH} / n$-hexane, 1:99, flow: $\left.1.0 \mathrm{~mL} \cdot \mathrm{min}^{-1}\right)$. $t_{\mathrm{r}}$ (major) $=5.0 \mathrm{~min}, t_{\mathrm{r}}$ (minor) $=4.2$ min. $[\alpha]_{\mathrm{D}}{ }^{25}+63.2\left(c=0.200\right.$ in $\mathrm{CH}_{2} \mathrm{Cl}_{2}, 97 \%$ ee $)$. [lit. $[\alpha]_{\mathrm{D}}{ }^{25}-61.9\left(c=1.0\right.$ in $\mathrm{CHCl}_{3}, 96 \%$ ee $)$; Fuerst, D. E.; Jacobsen, E. N. J. Am. Chem. Soc. 2005, 127, 8964-8965.
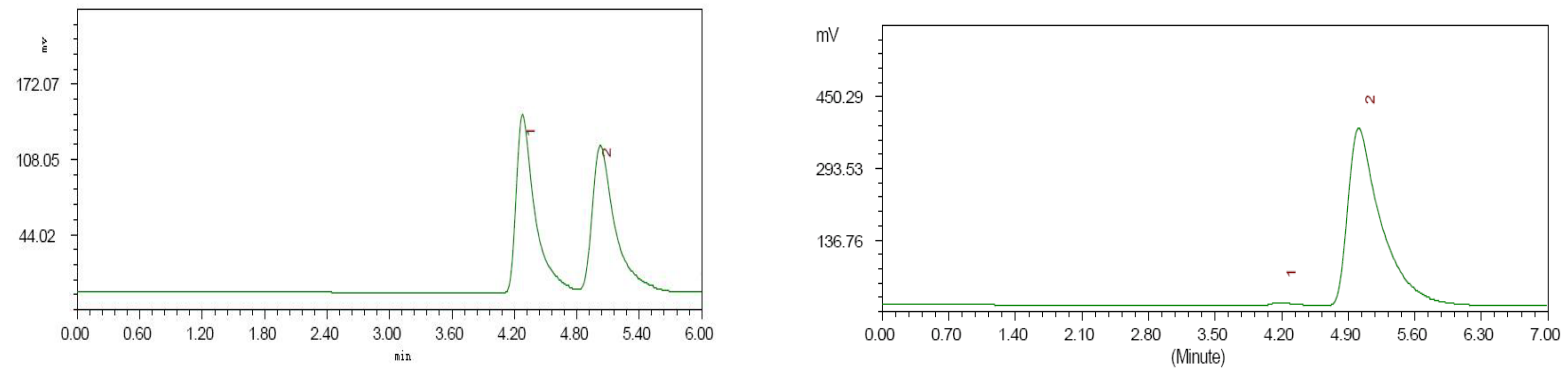

\section{2-trimethylsilyloxy-2(2'-naphthyl)propanenitrile (3j)}

90\% yield, $96 \%$ ee. ${ }^{1} \mathrm{H}$ NMR $\left(300 \mathrm{MHz}, \mathrm{CDCl}_{3}\right): \delta=0.22(\mathrm{~s}, 9 \mathrm{H}), 1.97(\mathrm{~s}, 3 \mathrm{H}), 7.54-7.66(\mathrm{~m}, 3 \mathrm{H}), 7.90-7.93(\mathrm{~m}, 3 \mathrm{H})$, $8.07(\mathrm{~d}, J=1.8 \mathrm{~Hz}, 1 \mathrm{H}) \mathrm{ppm}$. HPLC (Chiralcel OJ, $i \mathrm{PrOH} / n$-hexane, 0.5:99.5, flow: $\left.0.5 \mathrm{~mL} \cdot \mathrm{min}^{-1}\right): t_{\mathrm{r}}($ major$)=10.3$ $\min , t_{\mathrm{r}}($ minor $)=14.7 \mathrm{~min} .[\alpha]_{\mathrm{D}}{ }^{25}+14.4\left(c=0.194\right.$ in $\mathrm{CH}_{2} \mathrm{Cl}_{2}, 96 \%$ ee $)$. [lit. $[\alpha]_{\mathrm{D}}^{20}-14.2\left(c=1.0\right.$ in $\mathrm{CHCl}_{3}, 97 \%$ ee $\left.)\right]$; Fuerst, D. E.; Jacobsen, E. N. J. Am. Chem. Soc. 2005, 127, 8964-8965.
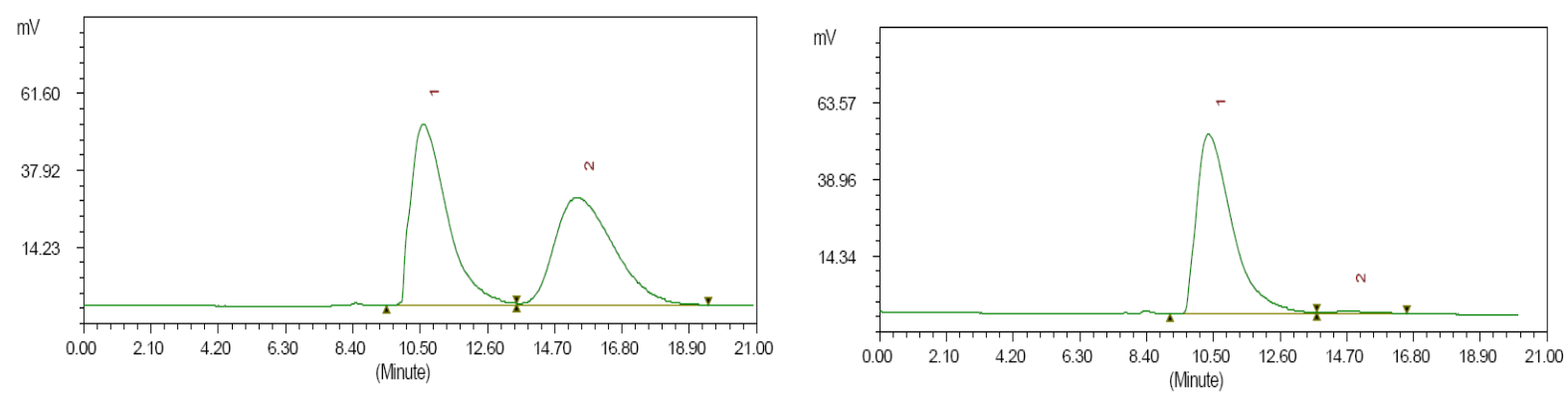

\section{2-trimethylsilyloxy- 2-methyl-4-phenyl-butanenitrile (3k)}

97\% yield, $81 \%$ ee. ${ }^{1} \mathrm{H}$ NMR (300 MHz, $\left.\mathrm{CDCl}_{3}\right): \delta=0.29(\mathrm{~s}, 9 \mathrm{H}), 1.65(\mathrm{~s}, 3 \mathrm{H}), 2.02-2.08(\mathrm{~m}, 2 \mathrm{H}), 2.80-2.91(\mathrm{M}, 2 \mathrm{H})$, 7.22-7.33 (m, 5H) ppm. GC analysis (Varian, CP-Chirasil DEX CB, 0.25mm x 25m ,130 ${ }^{\circ} \mathrm{C}, 8$ psi): $t_{\mathrm{r}}$ (major) $=31.5$ $\min , t_{\mathrm{r}}($ minor $)=32.1$ min. $[\alpha]_{\mathrm{D}}{ }^{25}+12.7\left(c=0.248\right.$ in $\mathrm{CH}_{2} \mathrm{Cl}_{2}, 81 \%$ ee $)$. [lit. $[\alpha]_{\mathrm{D}}{ }^{25}+13.3\left(c=1.15\right.$ in $\mathrm{CHCl}_{3}, 81 \%$ ee $\left.)\right]$; Hamashima, Y.; Kanai, M.; Shibasaki, M. J. Am. Chem. Soc. 2000, 122, 7412-7413 

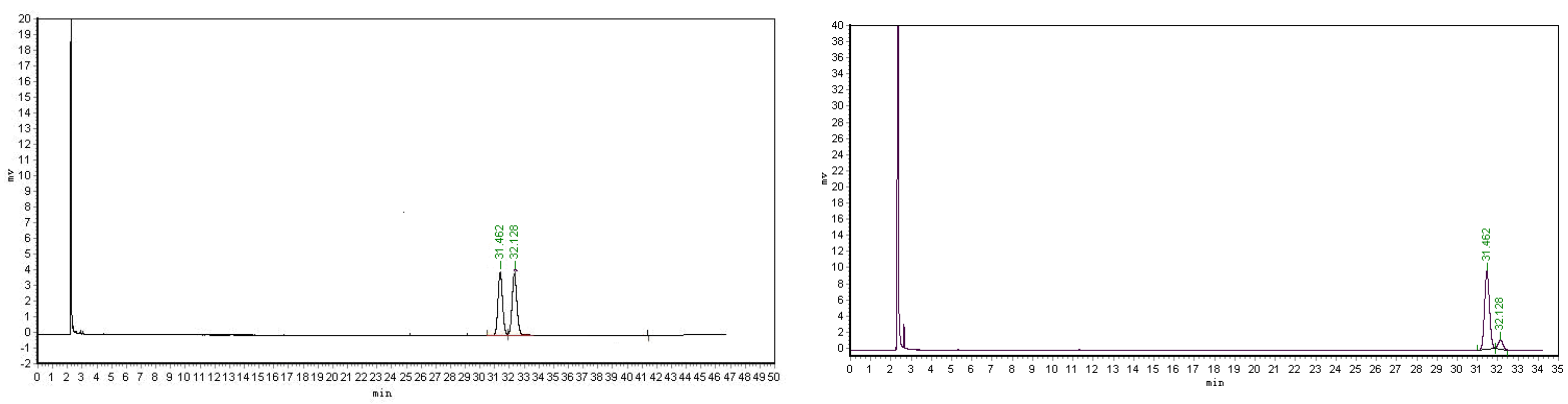

\section{2-trimethylsilyloxy-2-methyl-3-methylbutanenitrile (3I)}

$92 \%$ yield, 55\% ee. ${ }^{1} \mathrm{H}$ NMR $\left(600 \mathrm{MHz}, \mathrm{CDCl}_{3}\right): \delta=0.24(\mathrm{~s}, 9 \mathrm{H}), 1.03(\mathrm{t}, J=6 \mathrm{~Hz}, 6 \mathrm{H}), 1.53(\mathrm{~s}, 3 \mathrm{H}), 1.86($ hept, $\mathrm{J}=$ $6 \mathrm{~Hz}, 1 \mathrm{H}$ ) ppm. GC analysis (Varian, CP-Chirasil DEX CB, 0.25mm x $25 \mathrm{~m}, 65{ }^{\circ} \mathrm{C}, 8 \mathrm{psi}$ ): $t_{\mathrm{r}}$ (major) $=20.8 \mathrm{~min}, t_{\mathrm{r}}$ (minor) $=21.5$ min. $[\alpha]_{\mathrm{D}}^{25}+1.2\left(c=0.170\right.$ in $\mathrm{CH}_{2} \mathrm{Cl}_{2}, 55 \%$ ee $)$. [lit. $[\alpha]_{\mathrm{D}}^{22}-1.1\left(c=1.66\right.$ in $\mathrm{CH}_{2} \mathrm{Cl}_{2}, 90 \%$ ee $\left.)\right]$; Chen, $\mathrm{F}$. X.; Zhou, H.; Liu, X. H.; Qin, B.; Feng, X. M. Chem. Eur. J. 2004, 10, 4790-4797.
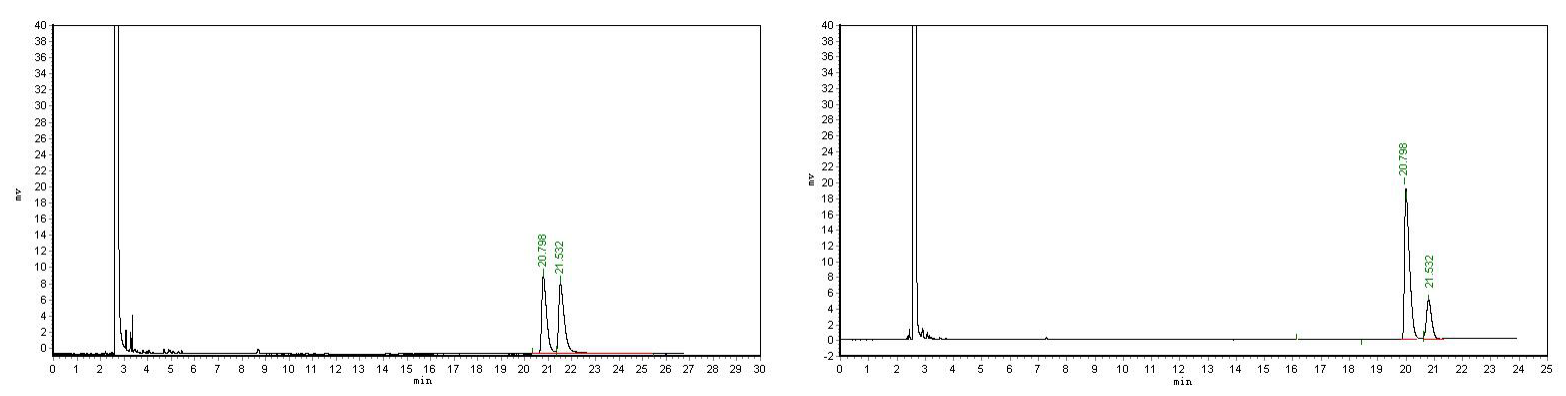

\section{Copy of NMR Spectra of TMSCN and the Catalyst}

When a 1:1 mixture of TMSCN and catalyst $2 \mathbf{f}$ in $\mathrm{CDCl}_{3}$ at $25^{\circ} \mathrm{C}$ was monitored by ${ }^{1} \mathrm{H} \mathrm{NMR}$, the development of new $\mathrm{CH}_{3}$ peak of TMSCN at $\delta=0.21 \mathrm{ppm}$ was observed $\left(\mathrm{CH}_{3}\right.$ of TMSCN peak at $\left.\delta=0.37 \mathrm{ppm}\right)$. The ${ }^{13} \mathrm{C}$ NMR spectra also changed, with a new $\mathrm{CH}_{3}$ peak appearing at $\delta=1.91 \mathrm{ppm}\left(\mathrm{CH}_{3}\right.$ of TMSCN peak at $\left.\delta=-1.83 \mathrm{ppm}\right) .{ }^{29} \mathrm{Si}$ NMR analysis revealed that another strong TMS singal was found at $\delta=0.6 \mathrm{ppm}$ as compared to $-17.9 \mathrm{ppm}$ for TMSCN 


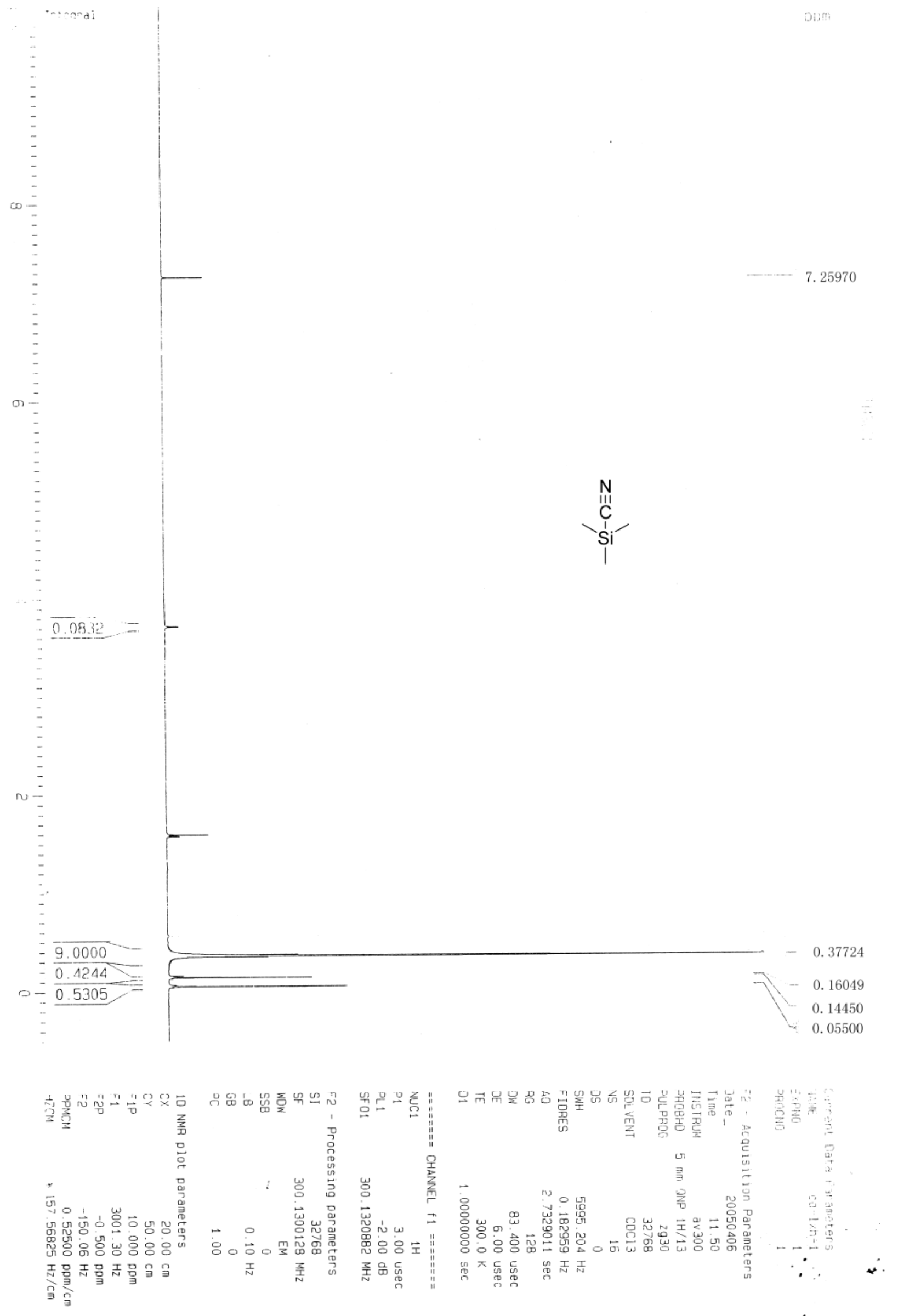




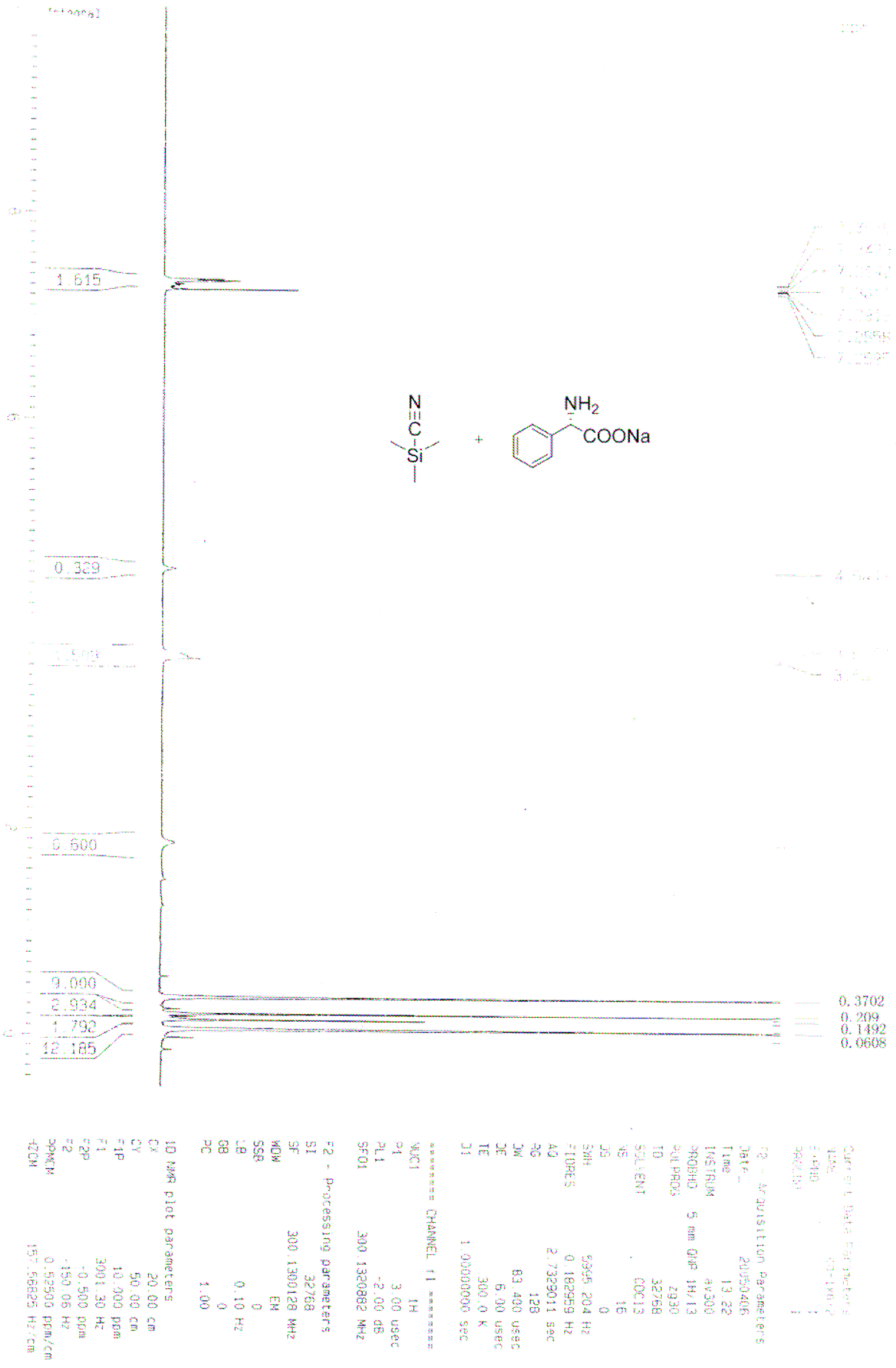



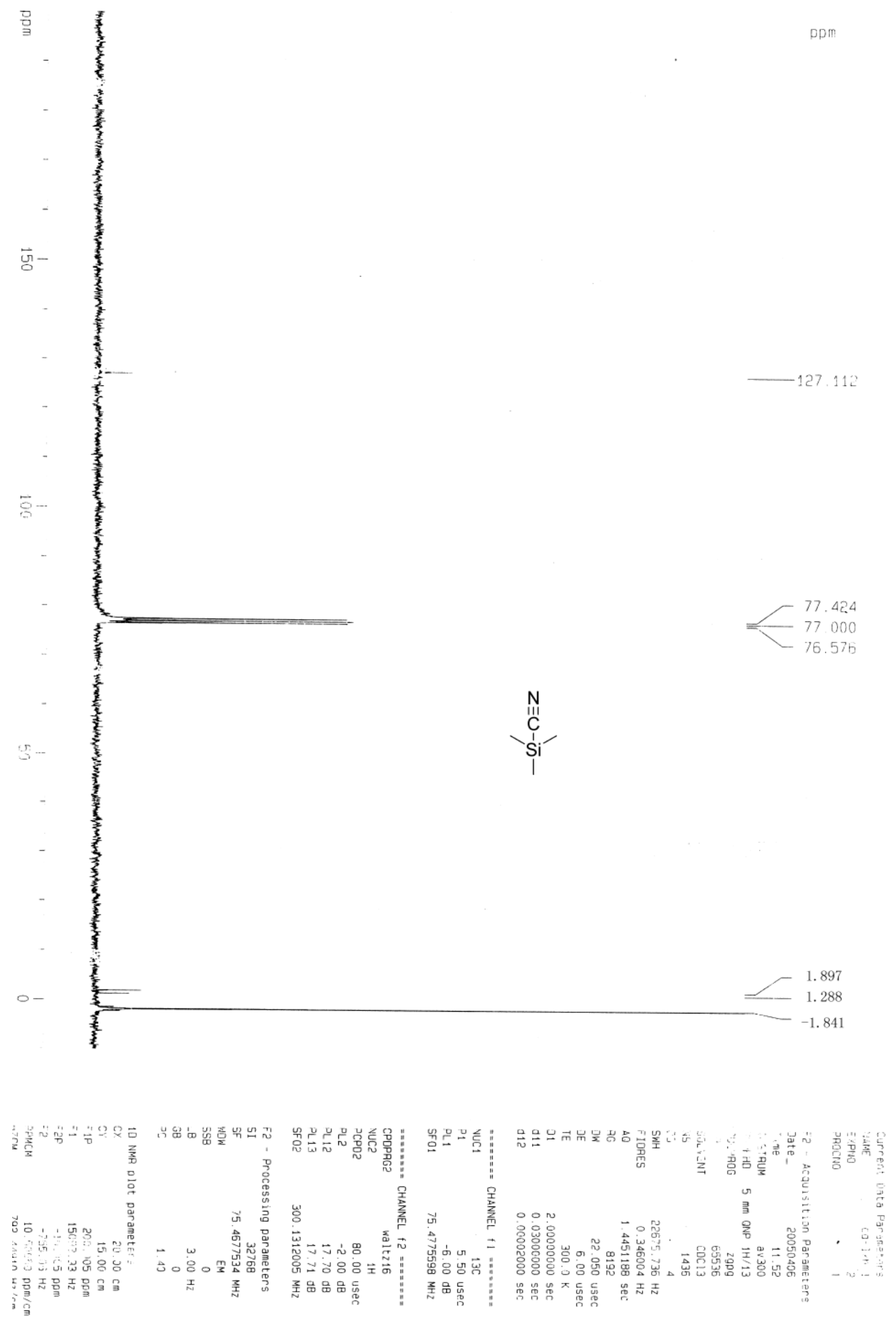

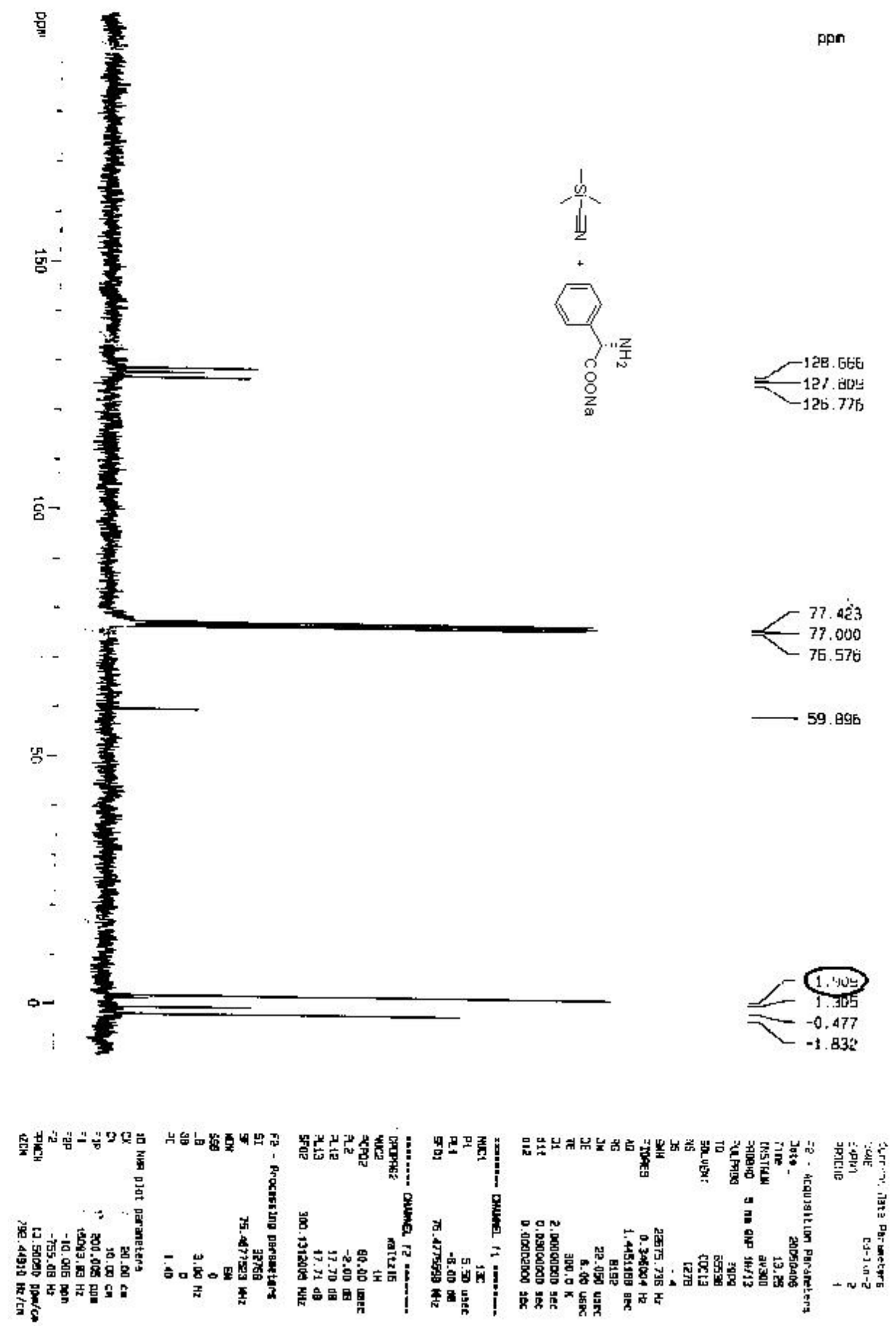


$$
1
$$




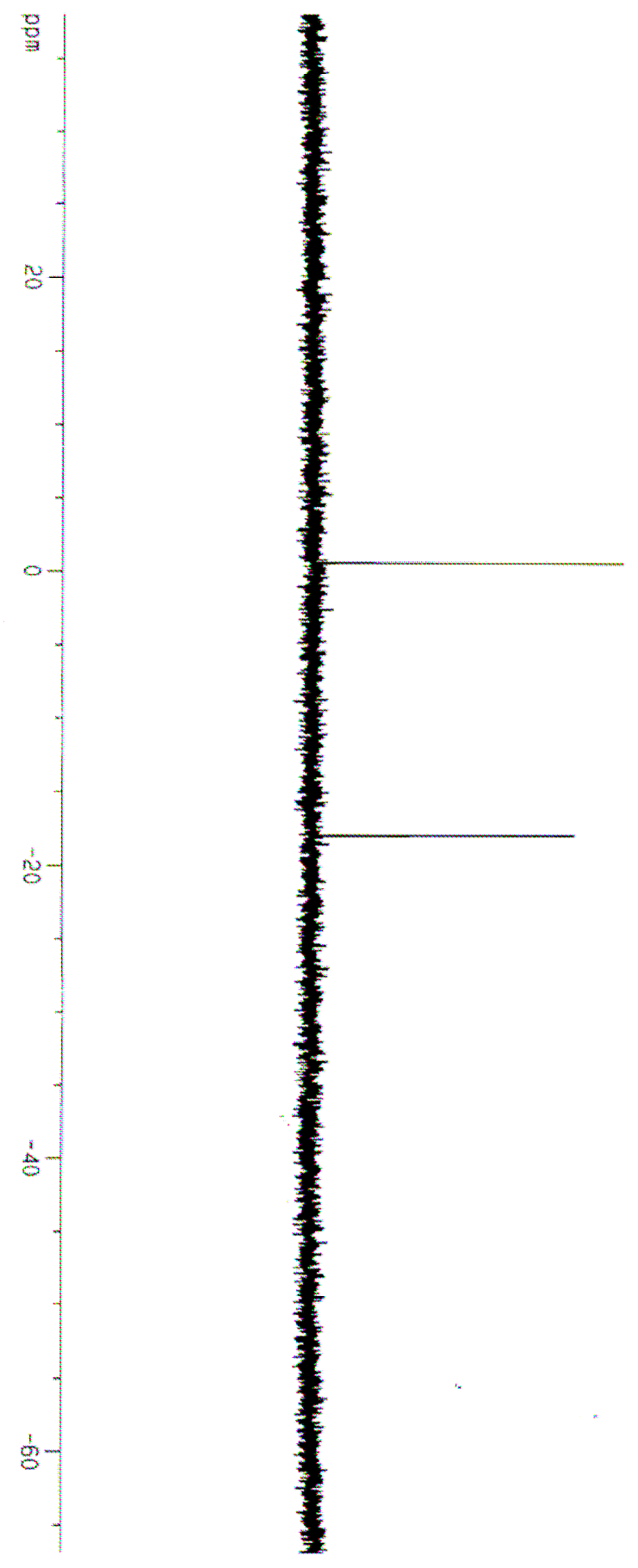

pom
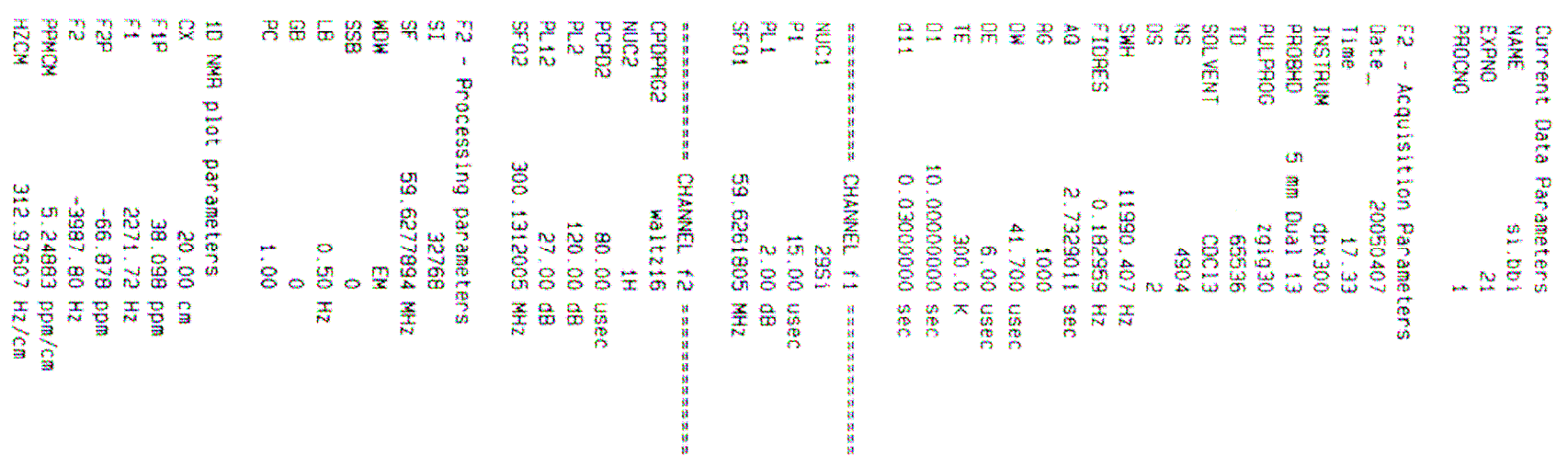